\title{
Anforderungen an die Vertretung in einer Gemeinschaftspraxis und Berechnung des Dreimonatszeitraums
}

\section{Einleitung}

Besonders in der Urlaubszeit, aber immer auch dann, wenn sich ein Vertragsarzt aus anderen Gründen bei seiner Tätigkeit vertreten lässt und seine vertragsärztliche Leistung nicht persönlich erbringt, ist Vorsicht geboten. Etwaige Verstöße gegen vertragsärztliche Vertretungsregelungen und den Grundsatz der persönlichen Leistungserbringung können im Rahmen einer anschließenden Rechtmäßigkeits- und Plausibilitätsprüfung zu einem Regress der Kassenärztlichen Vereinigung (KV) und einer Rückforderung des Honorars führen. Dies gilt unabhängig von der Art der geführten Praxis für Praxisgemeinschaften, Berufsausübungsgemeinschaften und Medizinische Versorgungszentren gleichermaßen. Das Sozialgericht München hatte in einer Entscheidung vom 20.01.2017 (Az.: S 28 KA 698/15) über die Folgen nicht ordnungsgemäßer Abwesenheitsmeldungen und die Rechtmäßigkeit von Honorarbescheiden bei der Vertretung innerhalb einer fachübergreifenden Gemeinschaftspraxis zu befinden. Darin konkretisierte das Gericht die Vertretungsregelung des $\S 32$ Ärzte-ZV im Zusammenhang mit der Bindung an die Grenzen des Fachgebiets und Versorgungsbereichs, sowie der Berechnung des Dreimonatszeitraums gemäß Absatz 1 Satz 2.

\section{Sachverhalt}

Die Beteiligten stritten um die Rechtmäßigkeit der Rücknahme und Neufestsetzung von Honorarbescheiden, die zu einer Rückforderung wegen eines Verstoßes gegen vertragsärztliche Vertretungsregelungen und gegen den Grundsatz der persönlichen Leistungserbringung führten. Die Klägerin war eine von zwei Vertragsärzten betriebene Gemeinschaftspraxis. Ein Arzt war Facharzt für Radiologie und war entsprechend zur vertragsärztlichen Versorgung zugelassen. Der andere war Facharzt für Radiologie und Nuklearmedizin, verfügte aber nur als Nuklearmediziner über einen vertragsärztlichen Versorgungsauftrag. Der als Facharzt für Radiologie zugelassene Arzt unternahm regelmäßige Fernreisen, die er auf seiner eigenen Homepage dokumentierte. Nachdem eine anonyme Anzeige bei der Stelle zur Bekämpfung von Fehlverhalten im Gesundheitswesen einging, stellte die KV aufgrund der Dokumentation des Arztes im Internet fest, dass dieser seine Abwesenheiten nicht, jedenfalls nur unvollständig der KV gemeldet hatte und er in den gemeldeten Abwesenheiten seinen Praxispartner als Vertreter angab. In Folge der Abrechnungsprüfung setzte die KV das Honorar für die in den Vertretungszeiträumen liegende Quartale neu fest und erließ einen Rückforderungsbescheid. Hiergegen wandte sich die klagende Gemeinschaftspraxis.

\section{Honorarrisiko des Arztes bei grob fahrlässig unrichtigen Abrechnungs- sammelerklärungen}

Zunächst stellte das Gericht fest, dass die sachlich-rechnerische Richtigstellung der beklagten KV auf Grundlage der Plausibilitätskontrolle gemäß §106a Abs. 1, Abs. 2 Satz 1 SGB V erfolgte, wonach KV und Krankenkassen die Rechtmäßigkeit und Plausibilität der Abrechnungen in der vertragsärztlichen Versorgung prüfen. Das Gericht erinnerte in diesem Zusammenhang an die Rechtsprechung des Bundessozialgerichts ${ }^{1}$, wonach bei einer (zumindest) grob fahrlässig unrichtigen Abrechnungssammelerklärung des Arztes für die KV grundsätzlich die Verpflichtung entfällt, als Voraussetzung der Rechtswidrigkeit des Honorarbescheides dem Arzt mehr als eine unrichtige Abrechnung pro Quartal nachzuweisen. Demnach ist sie rechtlich nicht verpflichtet, in allen Behandlungsfällen, in denen sie unrichtige Abrechnungen vermutet, den Nachweis der Unrichtigkeit zu führen. Entsprechend liegt das Honorarrisiko letztlich immer auf Seiten des Arztes, der unrichtige

1 Bundessozialgericht, Urteil vom 17.09.1997, Az.: 6 RKa 86/95, Rn. 21.

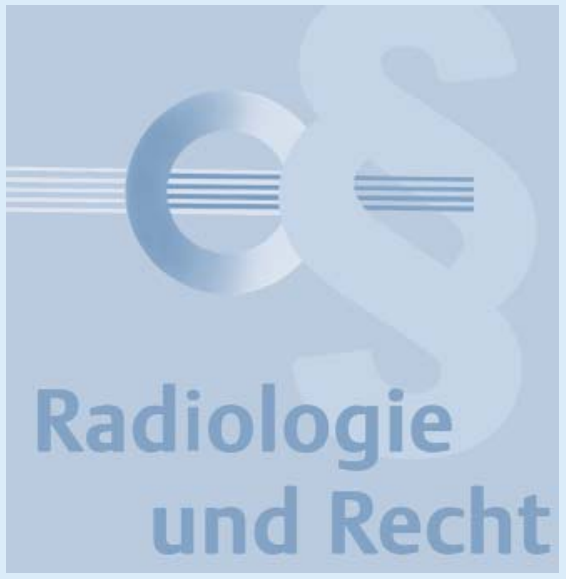

Angaben in seiner Abrechnung gemacht hat. Dies bedeutet, dass bereits bei Vorliegen einer einzelnen grob fahrlässig falschen Angabe auf einem Behandlungsausweis der Honorarbescheid für das betreffende Quartal im Ganzen fehlerhaft ist. Dies gilt auch und vor allem für nicht oder nur unvollständig der KV gemeldete Abwesenheiten des Vertragsarztes. Nach Aufhebung des unrichtigen Honorarbescheides setzt die KV das der Gemeinschaftspraxis zustehende Honorar neu fest, soweit diese die Leistungen tatsächlich und ordnungsgemäß erbracht hatte. Bei der Festsetzung hat die KV ein weites, jedoch gerichtlich vollständig überprüfbares Schätzungsermessen², so dass das Sozialgericht die Schätzung nachvollziehen können muss oder sie selbst vornehmen kann.

\section{Grundsatz der persönlichen Leistungserbringung gemäß $\S 32$ Abs. 1 Satz 1 Ärzte-ZV}

Die Pflicht des Arztes zur persönlichen Leistungserbringung, als eines der wesentlichen Merkmale der freiberuflichen Tätigkeit, erfordert, dass der Arzt auch bei Erbringung eigener beruflicher Leistungen durch nichtärztliche und ärztliche Mitarbeiter zumindest leitend und eigenverantwortlich tätig wird. Dementsprechend hat der Vertragsarzt gemäß $§ 32$ Abs. 1 Satz 1 Ärzte-ZV die vertragsärztliche Tätigkeit persönlich in freier Praxis auszuüben. Hierbei kann er sich nach § 32 Abs. 1 Satz 2 Ärzte-

2 Bundessozialgericht, Urteil vom 17.09.1997, Az.: 6 RKa 86/95, Rn. 23. 
ZV bei Krankheit, Urlaub oder der Teilnahme an einer ärztlichen Fortbildung oder Wehrübung innerhalb von zwölf Monaten bis zu einer Dauer von drei Monaten vertreten lassen. Die Vertretung bedarf grundsätzlich keiner Genehmigung. Soweit diese jedoch länger als eine Woche dauert, besteht für den Vertragsarzt gemäß $\S 32$ Abs. 1 Satz 4 Ärzte-ZV eine Mitteilungspflicht gegenüber der KV. Der Vertragsarzt ist verpflichtet, für eine geeignete ärztliche Vertretung Sorge zu tragen, sofern er seiner persönlichen Tätigkeitsverpflichtung nicht nachkommen kann.

\section{Bindung an die Grenzen des Fachgebiets und Versorgungsbereichs im Rahmen einer fachübergreifenden Gemeinschaftspraxis}

Nach Auffassung des Sozialgerichts München, die auf einer Entscheidung des Bundessozialgerichts ${ }^{3}$ beruht, findet die Vorschrift des §32 Ärzte-ZV auf die Klägerin als fachübergreifende Gemeinschaftspraxis Anwendung, weil der eine Vertragsarzt nicht den Ausfall des jeweils anderen Arztes auffangen konnte. Soweit sich der abwesende Facharzt für Radiologie während seiner Fernreisen umfassend durch seinen Praxispartner vertreten ließ, hätte Letzterer als Facharzt für Nuklearmedizin nicht das gesamte Spektrum der vertragsärztlichen Tätigkeit des Vertretenen abdecken dürfen. Für ihn als Facharzt für Nuklearmedizin seien Röntgen- und CT-Leistungen fachfremde Leistungen ${ }^{4}$, so dass der Vertretung Abrechnungsbestimmungen des Einheitlichen Bewertungsmaßstabes entgegenstünden ${ }^{5}$. Für eine ordnungsgemäße Vertretung hätte es eines praxisexternen Vertreters bedurft, der seinerseits als Facharzt für Radiologie zur vertragsärztlichen Versorgungstätigkeit zugelassen ist. Alle von dem Vertreter erbrachten Leistungen seien nach Auffassung des Sozialgerichts fehlerhaft abgerechnet worden und konnten daher durch die KV zurückgefordert werden.

Diese rechtliche Bewertung des Sozialgerichts München ist kritisch zu hinterfragen. Richtig ist zwar, dass die Grenzen des Fachgebiets und des Versorgungsbereichs auch für Ärzte gelten, die in einer fachgebietsund versorgungsübergreifenden Gemeinschaftspraxis tätig sind. Entsprechend des $\S 20$ Abs. 1 Satz 2 Musterberufsordnung dürfen sich Ärztinnen und Ärzte nur durch eine Fachärztin oder einen Facharzt desselben Fachgebiets vertreten lassen. Dies soll nach zutreffender Auffassung des Bundessozialgerichts auch für die Vertretung der Partner in einer Gemeinschaftspraxis gelten. Im Unterschied zum vorliegenden Sachverhalt wollte in dem der Entscheidung des Bundessozialgerichts zugrunde liegenden Sachverhalt ein Hausarzt EBMLeistungen abrechnen, die allein Fachärzten für Innere Medizin vorbehalten sind ${ }^{6}$. Ein solcher Fall fachgebietsfremder EBM-Leistungen lag hier jedoch nicht vor. Eine derartige Einschränkung besteht zwar für die Berechnung der Leistungen der Nuklearmediziner, umgekehrt aber nicht für die der Radiologen. Insoweit hätte das Sozialgericht München der Frage nachgehen müssen, ob der Nuklearmediziner als Doppelfacharzt in diesem Fall als externer Vertreter zu betrachten gewesen wäre. Darüber hinaus hätte sich das Sozialgericht München in seiner Entscheidung auf den Standpunkt stellen können, dass der Nuklearmediziner als Doppelfacharzt zunächst seinen eigenen Versorgungsauftrag selbst hätte erfüllen müssen, um anschließend zu prüfen, welche Leistungen er daneben zusätzlich noch als externer Vertreter und nicht zugelassener Radiologe erbringen durfte. Beide Fragen lässt das Urteil des Sozialgerichts München unberücksichtigt.

6 Streitgegenständlich war die Berechnung der Gebührenposition Nr. 13400 EBM-Ä, vgl. BSG Urt. v. 14.12.2011 - B 6 KA 31/10 R, Rn. 12.

\section{Berechnung der Tätigkeitsdauer von drei Monaten}

Das Sozialgericht München stellte zudem klar, dass es bereits in der Vergangenheit der überwiegenden herrschenden Meinung entsprach, dass die Dreimonatsfrist nicht durch Addition der Vertretertage sondern fortlaufend von dem Zeitpunkt an zu errechnen sei, an dem der Vertreter erstmalig tätig wird ${ }^{7}$. Dies entspräche auch der Auffassung des Bundessozialgerichts, wonach bereits aus dem Wortlaut der Vorschrift hervorgehe, dass es nicht auf die einzelnen Tage ankomme, an denen der Vertreter tätig werde, sondern auf den Zeitraum, in dem die Vertretung aus den in $\S 32$ Abs. 1 Ärzte-ZV genannten Gründen erforderlich ist $^{8}$. Zudem folge dies aus dem Sinn der Regelung, wonach der an der vertragsärztlichen Versorgung teilnehmende Arzt zur vertragsärztlichen Versorgung nicht nur berechtigt, sondern auch verpflichtet wäre ${ }^{9}$. Entsprechend des $\S 32$ Abs. 1 Satz 2 ÄrzteZV könne der in freier Praxis seine Tätigkeit ausübende Vertragsarzt, sich als Ausnahme vom Grundsatz der persönlichen Leistungserbringung bei einer Verhinderung vertreten lassen und gleichwohl diese Leistungen gegenüber der KV wie eigene abrechnen, ohne dass es hierfür einer Genehmigung bedürfe. Auch wenn der Vertragsarzt seiner Verpflichtung zur Teilnahme an der vertragsärztlichen Versorgung nur ungenügend nachkomme, indem er sich in einem geringeren Umfang vertreten ließe, könne dies aber nach dem Sinn der Regelung nicht zu einer Verlängerung des Zeitraums der genehmigungsfreien Vertretung führen.

Demnach könne es auch nicht auf die Anzahl der Vertretertage ankommen, sondern allein auf den Zeitraum, in dem der Vertragsarzt abwesend war und zwar von dem Zeitpunkt an, an dem der Vertreter erstma-

7 Bäune/Meschke/Rothfuß, Kommentar zur Zulassungsverordnung für Vertragsärzte und Vertragszahnärzte, 2008, §32, Rn. 18

8 Bundessozialgericht, Beschluss vom 13.08.2014, Az.: B 6 KA 3/14 B, Rn. 6 zum gleichlautenden $\S 32$ Abs. 1 Satz 2 ZahnärzteZV.

9 Dies geht aus den Vorschriften der $\S \S 72$ Abs. 1 Satz 2, 95 Abs. 3 Satz 1 SGB V hervor. 
lig tätig wurde. In den Fällen, in denen der erste Abwesenheitstag nicht zugleich der erste Vertretertag ist, berechnet sich der Zeitraum erst ab dem Tag, an dem der Vertreter erstmals tätig wurde. Sofern beispielsweise der Urlaub des Vertretenen an einem Wochenende oder an einem Feiertag beginnt und die Praxis Sprechzeiten von Montag bis Freitag anbietet, ist eine Vertretung über die Sprechzeitentage hinaus nicht erforderlich. Für einen Urlaub von Samstag bis Samstag müssten demnach fünf Vertretungstage einberechnet werden.

\section{Fazit}

Das Honorarrisiko bei (zumindest) grob fahrlässig unrichtigen Abrechnungssammelerklärungen liegt immer bei dem abrechnenden Vertragsarzt. Hierdurch entfällt für die KV grundsätzlich die Verpflichtung, als Voraussetzung der Fehlerhaftigkeit des Honorarbescheides dem Arzt mehr als eine unrichtige Abrechnung pro Quartal nachzu- weisen, so dass bereits bei Vorliegen einer einzelnen grob fahrlässig falschen Angabe auf einem Behandlungsausweis der Honorarbescheid für das betreffende Quartal im Ganzen fehlerhaft ist. Dies sollte im Zusammenhang mit einer unvollständigen Abgabe ärztlicher Abwesenheitszeiten gegenüber der Kassenärztlichen Vereinigung stets bedacht werden. Prüfen Kassenärztliche Vereinigungen und Krankenkassen die Rechtmäßigkeit und Plausibilität der Abrechnungen in der vertragsärztlichen Versorgung im Zusammenhang mit einer Vertretung des Vertragsarztes, ist eine gegenseitige Vertretung der Vertragsärzte in einer fachübergreifenden Gemeinschaftspraxis wegen der Bindung an die Grenzen des Fachgebiets grundsätzlich nicht möglich. Dies gilt umso mehr, wenn der Vertretung Abrechnungsbestimmungen des Einheitlichen Bewertungsmaßstabes entgegenstehen, die eine Abrechnung fachfremder Leistungen nicht zulassen. Schließlich ist der Dreimonatszeitraum gemäß $\S 32$ Abs. 1 Satz 2 Ärzte-ZV nicht durch eine Ad- dition der Vertretertage, sondern fortlaufend von dem Zeitpunkt an zu errechnen, an dem der Vertreter eines Vertragsarztes erstmalig tätig wird. Ist der erste Abwesenheitstag nicht zugleich der erste Vertretertag, berechnet sich der Zeitraum erst ab dem Tag, an dem der Vertreter erstmals tätig werden musste. Zu den Vertretungstagen sind die Vertretungen für Bereitschaftsdienste ebenfalls hinzuzurechnen.

Prof. Dr. Peter Wigge

Rechtsanwalt

Fachanwalt für Medizinrecht

Dr. Nikolaus R. Marek, Rechtsanwalt

Rechtsanwälte Wigge

Scharnhorststraße 40

48151 Münster

Telefon: (0251) 53595 - 0

Telefax: (0251) 53595 - 99

E-mail: kanzlei@ra-wigge.de http://www.ra-wigge.de 